\title{
Vibroequilibria in microgravity: Comparison of experiments and theory
}

\author{
P. Salgado Sánchez $\odot$, J. Fernández $\odot$, I. Tinao, and J. Porter@ \\ Center for Computational Simulation, Escuela Técnica Superior de Ingeniería Aeronáutica y del Espacio, \\ Universidad Politécnica de Madrid, Plaza de Cardenal Cisneros 3,28040 Madrid, Spain
}

\begin{abstract}
Experiments on vibrated fluids confined in cylindrical and cuboidal containers were performed under the reduced gravity conditions of a parabolic flight. The results constitute a systematic quantitative investigation of the vibroequilibria effect, which refers to the reorientation of vibrated fluids in response to the inhomogeneous oscillatory velocity field and the accompanying dynamic pressure. This effect is amplified in microgravity where the restoring force of gravity is small or absent. Here the vibrations are transmitted via a pair of piezoelectric ceramics and a cantilever beam, excited in a resonant mode. The first and second resonances exhibit different types of motion and lead to different types of vibroequilibria surfaces, one with a dip or crater in the interior and the other flattened compared to the unforced reference experiment. The general tendency for interfaces to orient more perpendicular, on average, to the vibrational axis is confirmed. In the case of water in a cuboidal container, a quantitative comparison is made with vibroequilibria theory and with direct simulations of the Navier-Stokes equations. The good agreement confirms the predictions of vibroequilibria theory and suggests the capacity of this phenomenon to manipulate and position fluids in space environments through the choice of frequency and resonant mode.
\end{abstract}

DOI: $10.1103 /$ PhysRevE.100.063103

\section{INTRODUCTION}

By nature, fluids move easily in response to external forces and the ability to manage and control this motion is a crucial aspect of wide-ranging applications in physics, chemistry, biology, medicine, and engineering. While small volumes of fluid may often be effectively manipulated using capillary effects, electromagnetic fields, or acoustic forces, this becomes increasingly difficult for larger volumes of liquid, whose distribution is dominated by gravity. Density stratification to minimize gravitational energy is a near-universal feature of fluid configurations larger than the capillary length scale and, among other things, explains the familiar sight of flat liquid-air interfaces on Earth.

In microgravity environments, on the other hand, fluid behavior is different. Surface tension and contact forces come to the fore and largely determine the shape and location of any free interfaces. A floating mass of liquid will minimize its surface energy by taking a spherical shape, while adhesive forces may cause it to wet a solid boundary on contact. The increased ease with which fluids can move (vertically) in microgravity, and the preference for curved hydrostatic interfaces rather than flat ones, means that fluid management is substantially more complicated than it is on Earth. At the same time, large-scale manipulation of fluids may become more feasible when gravity is no longer the limiting factor.

Vibrations are an inherent feature of most real environments, with their importance for a given physical process depending on amplitude, frequency, and orientation. Even in the simple case of purely periodic motion, they can affect a

\footnotetext{
"pablo.salgado@upm.es
}

range of important fluid phenomena such as mixing [1] and mass transfer.

If there is an interface separating fluids of different density, vibrations will generally excite waves. The vibrating motion of a solid support or boundary generates harmonic (synchronous) waves that decay, due to viscous effects, as they travel inward. At a critical amplitude, subharmonic waves (having twice the period of the vibrational forcing) may be excited via a parametric forcing mechanism arising from the oscillating pressure gradient [2,3]. If the source of this parametric forcing is localized, as it is near a side wall in a horizontally vibrating open container of liquid, then the subharmonic waves are similarly localized and are commonly known as cross-waves [4-7]. On the other hand, if the motion is vertical, perpendicular to the unperturbed flat interface, then the forcing is homogeneous and produces subharmonic Faraday waves [8-10] across the entire domain.

In addition to standing or traveling waves, vibrations parallel to an interface separating fluids of different density can drive a Kelvin-Helmholtz-type instability producing quasisteady, or "frozen," waves [11-13]. These frozen waves are initially sinusoidal in form and grow as the amplitude of vibrations increases. As with oscillatory disturbances like Faraday waves and cross-waves, they may be considered, in the usual supercritical case near onset, as perturbations of the initial flat interface. This is no longer so without the restoring force of gravity. In weightlessness, the frozen waves rapidly develop into large-scale columnar structures with alternating (nearly) vertical interfaces $[14,15]$. Recent microgravity experiments $[16,17]$ have shown that Faraday waves can subsequently be excited on these columnar interfaces to create a complex structure with two characteristic wavelengths. The pattern selection process is mainly controlled by vibrational velocity but is affected by viscosity [13] and finite-size effects [18]. 
The example of frozen waves illustrates the dramatic effect that even small-amplitude vibrations can have in microgravity. In contrast to the situation on ground, they can lead to a largescale redistribution of the liquids involved and, in so doing, facilitate a secondary Faraday wave instability that would not otherwise occur. It is not necessary to have the frozen wave (Kelvin-Helmholtz) instability for such a redistribution to happen either. The inhomogeneous oscillatory velocity field is naturally accompanied by an inhomogeneous dynamic pressure that drives a reorientation of the fluid. This phenomenon, known as the vibroequilibria effect, was observed by Faraday [8] in the flattening of drops beneath a vibrating plate. Ordinary gravity masks the vibroequilibria effect for most common fluid configurations (unless very large forcing is applied), while in microgravity environments, vibrations compete only with surface tension and contact forces so their effect is greatly enhanced.

The usual theoretical approach to vibroequilibria [19-21] assumes a separation of timescales between the highfrequency forcing and the slower viscous diffusion of momentum. If the flow is further assumed to be potential, then a variational method can be used to determine the quasisteady equilibrium of the surface as the critical point of a Lagrangian averaged over the fast timescale.

A recent investigation [22] compared vibroequilibria theory with direct numerical simulations and found good agreement over a range of parameters. It was further demonstrated that, depending on fluid depth, the symmetric vibroequilibria solution in a horizontally vibrated rectangular container can disappear in a saddle-node bifurcation, after which the surface dips violently toward the lower boundary and undergoes a disordered transition to one of many possible asymmetric states. In low-viscosity fluids, surface waves can interact with the underlying vibroequilibria state and may even destabilize it through excitation of the first odd sloshing mode; this is likely related to the presence of temporal modulations resulting from the interaction between subharmonic wave fields emanating from opposite sides of the container $[3,23,24]$.

The first experimental observation of the vibroequilibria effect in confined fluids was by Wolf [11], who applied largeamplitude vibrations $(936 \mathrm{~mm} / \mathrm{s}$ ) to a 60 - $\mathrm{mm}$-long cylindrical container of diameter $28 \mathrm{~mm}$ holding an aqueous solution of potassium iodide and a lighter oil (SAE 140). In microgravity, the only prior vibroequilibria experiment that we are aware of was conducted by Ganiev et al. [25], who investigated combinations of water, oil, and air in the reduced gravity of a parabolic flight. Partially filled cylinders of length $100 \mathrm{~mm}$ and diameter $30 \mathrm{~mm}$ were vibrated at low frequencies $(8 \mathrm{~Hz}$, $12 \mathrm{~Hz}$ ), producing disordered dynamics, and at high frequencies $(600-800 \mathrm{~Hz})$, where deep funnels or craters were seen for the air-water interface near a resonance frequency. This behavior, which is now recognized as indicative of the vibroequilibria effect in horizontally vibrated containers [22], was only later shown [21] to be qualitatively consistent with vibroequilibria theory and was not quantitatively compared with either theory or simulations.

A more systematic experimental investigation of vibroequilibria solutions and their importance in microgravity is called for, not only for the potential observation of novel states like those mentioned above, but for their ability to

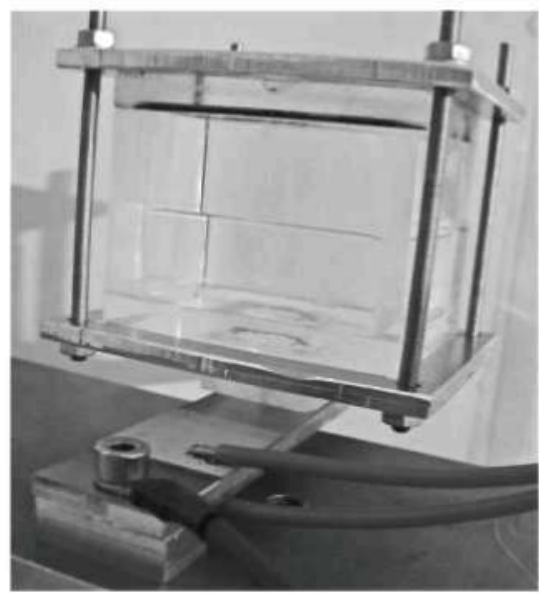

(a)

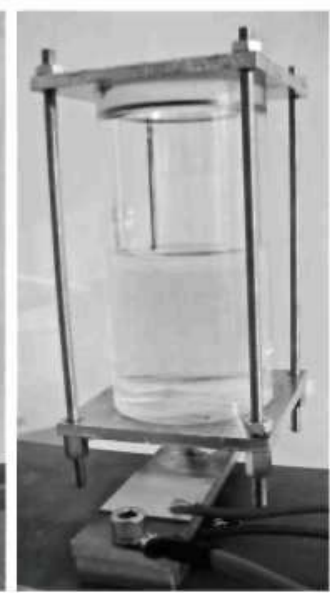

(b)
FIG. 1. Pictures of the experimental cells and the bender beam: (a) cuboid and (b) cylinder. The cells are held closed by four bolts, which compress the PMMA body and cover against an O-ring. The aluminum bender beam has two piezoelectric ceramics attached to opposite horizontal faces. Cables associated with electrical excitation of the ceramics and the clamping bolt can also be seen.

manipulate fluids in weightless environments. Indeed, vibrations are known to mimic the effect of gravity in certain aspects of convection and phase transitions [26] and vibroequilibria can be regarded in this light too. The general tendency, with increasing forcing, is for the vibroequilibria states to develop so that large portions of the surface are nearly perpendicular to the axis of vibration, reminiscent of how fluid surfaces align perpendicular to gravity.

With this motivation, the behavior of fluids in microgravity and the potential for controlling them through the vibroequilibria effect were investigated in the Control of Fluids in microgravity with Vibrations (CFVib) experiment [27], which looked at representative liquid-gas and two-liquid combinations in rectangular cuboidal and cylindrical containers excited by periodic vibrations and was performed during the 65th ESA Parabolic Flight campaign. The vibroequilibria effect was measured as a function of forcing amplitude at different resonance frequencies having different characteristic modes of vibration.

In this paper, we present the main scientific results obtained during the CFVib experiment and compare them to theoretical predictions. The experimental setup is described in Sec. II. The mathematical formulation used in the numerical simulations is given in Sec. III, together with a basic review of vibroequilibria theory. The most relevant results of the experiment are presented in Secs. IV and V, while the case of water in a cuboidal container is quantitatively compared both to direct numerical simulations and to vibroequilibria theory in Sec. V C. Conclusions are provided in Sec. VI.

\section{THE EXPERIMENT}

The experiment is designed to compare pairs of cuboidal and cylindrical cells (see Fig. 1) containing a liquid-air or immiscible two-liquid combination (the latter is not described here since the experimental results were inconclusive). One of 
TABLE I. Characteristic physical properties of the experimental liquids at room temperature; density $\rho$, surface tension $\Gamma$, and kinematic viscosity $v$. These values are used in the mathematical simulations using the formulation of Sec. III.

\begin{tabular}{rlll}
\hline \hline & $\rho\left(\mathrm{kg} / \mathrm{m}^{3}\right)$ & $\Gamma(\mathrm{N} / \mathrm{m})$ & $\nu\left(\mathrm{m}^{2} / \mathrm{s}\right)$ \\
\hline Water & 1000 & $7.20 \times 10^{-2}$ & $1 \times 10^{-6}$ \\
$50-\mathrm{cSt}$ silicone oil & 960 & $2.08 \times 10^{-2}$ & $50 \times 10^{-6}$ \\
\hline \hline
\end{tabular}

the cells is subjected to vibrations at various amplitudes and frequencies, while the reference cell is unforced and responds only to the residual acceleration present on the parabolic flight. These two simple geometries, which represent common fluid containers, can be expected to lead to different interfacial dynamics. In addition, the use of cuboidal cells has the advantage that (quasi) two-dimensional behavior can be assumed in some cases, while the cylindrical cells can be compared with the previous observations of Ganiev et al. [25].

The containers are made of transparent polymethyl methacrylate (PMMA) to allow for visualization of the liquid interface. The cuboids are $30 \mathrm{~mm} \times 40 \mathrm{~mm} \times 30 \mathrm{~mm}$ (length $\times$ width $\times$ height), while the cylinders have an interior radius of $15 \mathrm{~mm}$ and a height of $60 \mathrm{~mm}$. Each cell is composed of two PMMA pieces that are held against each other using bolted aluminum plates and sealed with an O-ring; part of this can be seen in Fig. 1. The results described here are obtained with water and 50-cSt silicone oil, whose relevant properties are given in Table $\mathrm{I}$.

Each experimental cell is fixed to an aluminum beam (cantilever) that, in the case of the excited cells, has two piezoelectric ceramics (PZTs), attached on opposite horizontal faces. An alternating counterphase voltage is applied to each PZT after being boosted by an amplifier. The counterphase mechanical stress induced in the ceramics creates a torque that drives periodic flexural motion of the beam and shakes the cell.

Both the amplitude $V$ (with a maximum determined by the amplifier) and frequency $f$ of the applied voltage can be varied to control the magnitude and characteristics of the vibrations. The experiments use voltages up to $100 \mathrm{~V}$, while the frequencies are selected to match the most relevant resonant modes of the assembly. The first and second resonances (at approximately 80 and $215 \mathrm{~Hz}$, respectively, for the cuboids) were found to be associated with the largest displacements and, therefore, the most pronounced vibroequilibria effects. Accelerometers directly measure the (vertical) acceleration on each vibrated container.

Although the motion of the container induced by the PZTs in a bender-beam configuration is not purely linear (see Sec. III for more details), combining translational motion with some degree of rotation, it has the advantage of small size and low power (compared to an electromagnetic shaker, for example). The selected transducer yields significantly higher amplitudes for a given voltage than alternative configurations (such as a biclamped beam), at least for the resonant modes excited here.

The optical system that monitors the position of the fluid includes a complementary metal-oxide-semiconductor

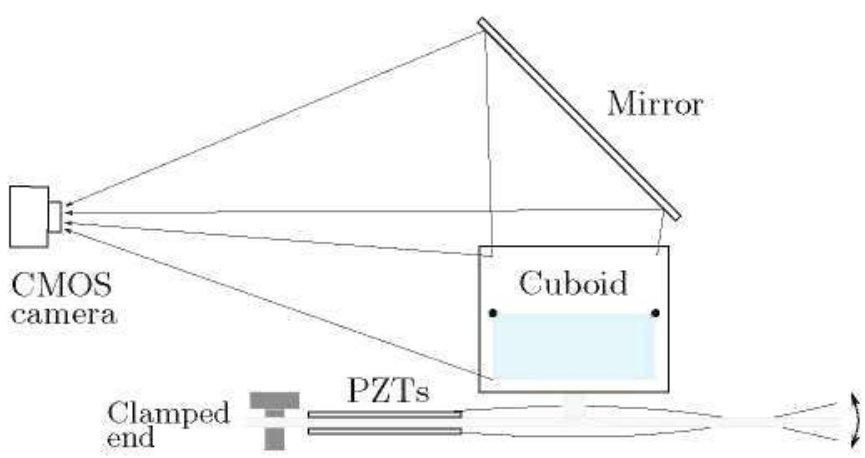

(a)

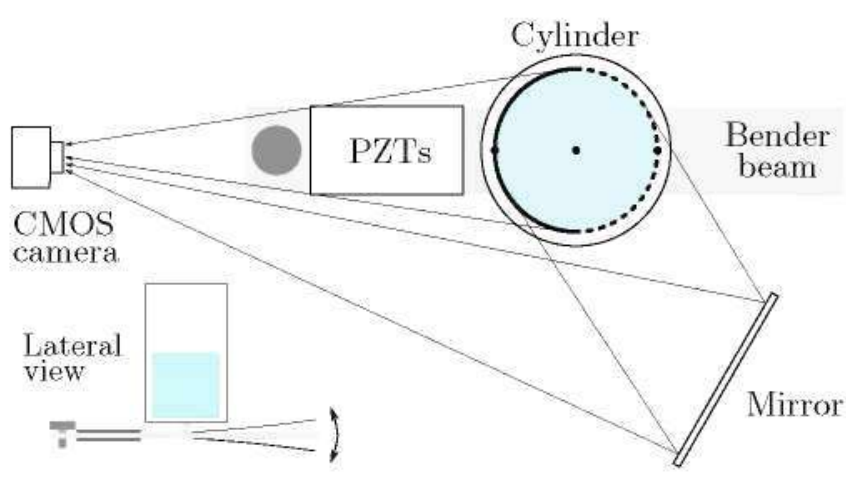

(b)

FIG. 2. Sketch of experimental setup with (a) cuboid and (b) cylinder. Shown are the (partially filled) experimental cell, bender beam, piezoelectric ceramics, mirror, and CMOS camera. Selected optical paths indicate the direct and reflected images referred to in later sections. The inset in (b) shows a lateral view of the setup for a cylindrical cell. Shaded curves illustrate the excited motion of the beam for the first ( $b$, inset) and second (a) resonant modes.

(CMOS) camera, a mirror, and a source of illumination. For the vibrated cells, the CMOS sensor samples at (approximately) 20 frames per second, providing excellent resolution of the slow timescale dynamics of the surface; the reference cells were recorded at (approximately) 10 frames per seconds. The behavior of the liquid surface was observed along the axis of the beam and after reflection by a mirror. Depending on the cell geometry, this mirror provides either a top view (for the cuboidal cell) or a lateral view (for the cylindrical cell). Both experimental setups are sketched in Fig. 2, which shows the container, bender beam, PZTs, CMOS camera, and mirror. Selected optical paths are drawn to illustrate the direct and reflected (top or lateral) views. Included illustrations indicate the dominant type of motion for the first resonant mode [see the inset in Fig. 2(b)] and the second one [Fig. 2(a)].

Experiments were performed under the reduced gravity provided by a parabolic flight [28], which lasts approximately $20 \mathrm{~s}$. The microgravity level (residual acceleration) during the parabolic maneuvers was measured by the onboard accelerometers and provided each day by the airplane operators [29]. This microgravity level satisfied

$$
\left|g_{\bar{z}}\right| \lesssim 0.05 g_{0}, \quad\left|g_{\tilde{x}, \bar{y}}\right| \lesssim 0.01 g_{0},
$$




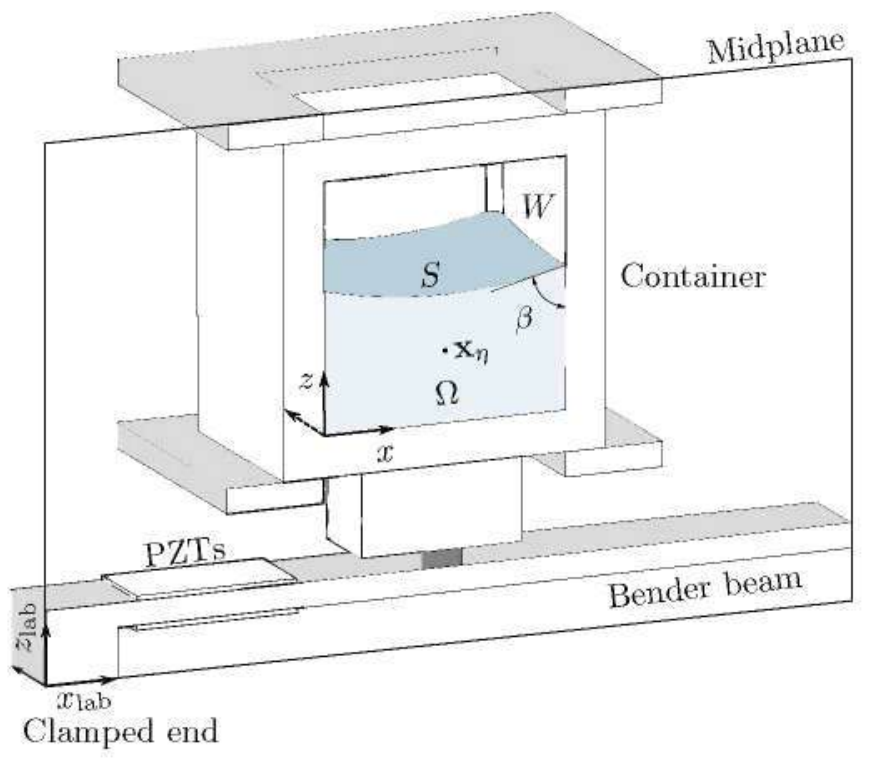

(a)

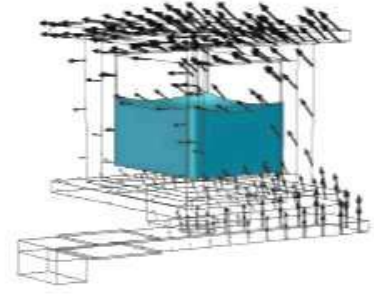

(b)

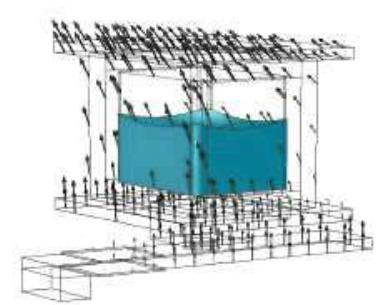

(c)
FIG. 3. (a) Midplane cut of the experimental setup for a cuboidal container showing the bender beam, PZTs, fluid volume $\Omega$, free surface $S$, and solid boundaries $W$. The container and laboratory reference frames are illustrated; since the displacement is very small compared to the container size, we assume hereafter that $(x, y, z) \simeq$ $\left(x_{\text {lab }}, y_{\text {lab }}, z_{\text {lab }}\right)$. This geometry is used for the numerical results shown in Sec. V C. The motion associated with the first two resonant modes is illustrated in (b) and (c), respectively.

where $g_{0}=9.81 \mathrm{~m} / \mathrm{s}^{2}$. The components are expressed in the airplane reference frame [28], defined by axes $\tilde{x}$, oriented aft to front; $\tilde{y}$, oriented along the wings; and $\tilde{z}$, perpendicular to the floor and identical to the vertical $z$ axis of the container (see Fig. 3). The cuboidal containers are aligned, approximately, with the airplane axes so that $(x, y) \approx(-\tilde{y}, \tilde{x})$, while the cylindrical containers are rotated by approximately $45^{\circ}$ (clockwise) with respect to the longitudinal axis of the plane.

In this paper, we highlight the results observed in microgravity with the cylindrical cells when vibrated in the first resonant mode and the cuboidal cells when vibrated in the first and second resonant modes. For further details about the experiment, see Ref. [27], where a review of the design, implementation, and execution is given.

\section{MATHEMATICAL FORMULATION}

We consider a finite volume of fluid $\Omega$ (see Fig. 3) with a free surface held in a container in the presence of a gravita- tional field and subjected to vibrations of frequency $\omega$. The fluid satisfies the incompressible Navier-Stokes momentum and mass continuity equations [30]

$$
\begin{aligned}
\mathbf{u}_{t}+(\mathbf{u} \cdot \nabla) \mathbf{u} & =-\nabla p+v \Delta \mathbf{u}+\mathbf{G}(\mathbf{x}, \mathbf{u}, t), \\
\nabla \cdot \mathbf{u} & =0,
\end{aligned}
$$

where $\mathbf{u}$ is the velocity, $\rho$ is the density, $v$ is the kinematic viscosity, and $p$ is the pressure.

The inhomogeneous acceleration $\mathbf{G}(\mathbf{x}, \mathbf{u}, t)$, which is expressed in the accelerating frame of the container, can be written, to leading order, as

$$
\begin{aligned}
\mathbf{G}(\mathbf{x}, \mathbf{u}, t)= & \mathbf{g}(t)+\omega \boldsymbol{v}_{\eta} \cos (\omega t)+2 \varpi \times \mathbf{u} \\
& +\varpi \times\left[\varpi \times\left(\mathbf{x}-\mathbf{x}_{\eta}\right)\right]+\dot{\boldsymbol{\omega}} \times\left(\mathbf{x}-\mathbf{x}_{\eta}\right),
\end{aligned}
$$

where $\mathbf{g}(t)$ is the time-dependent residual gravity field in the parabolic flight environment, $\boldsymbol{v}_{\eta}$ is the (approximately) linear velocity of a reference point $\mathbf{x}_{\eta}$, taken here to be the center of the unperturbed fluid (see Fig. 3), and $\varpi(t)$ is the rotational velocity of the container about that reference point. Both the linear and rotational motion are assumed to lie in the $(x, z)$ plane defined in Fig. 3. The magnitude of the rotational velocity $|\varpi(t)|=\varpi_{0} \sin (\omega t)$ depends on the mode of vibration and the forcing amplitude, but $\varpi_{0}$ is typically on the order of $1 \mathrm{rad} / \mathrm{s}$.

Navier-slip boundary conditions [18] are imposed on the solid boundaries $W$ to preserve the contact angle $\beta$ and allow for motion of the contact line. The balance among surface tension, pressure, and viscous stresses holds at the free surface $S$ defined by $f(\mathbf{x}, t)=0$,

$$
\begin{aligned}
\mathbf{u} \cdot \mathbf{n}_{w}=0 & \text { on } W, \\
p \mathbf{n}-\left(\frac{\Gamma}{\rho}\right) \kappa \mathbf{n}-v(\nabla \mathbf{u}) \cdot \mathbf{n}=0 & \text { on } S, \\
f_{t}+\mathbf{u} \cdot \nabla f=0 & \text { on } S .
\end{aligned}
$$

Here $\kappa$ is the mean curvature of the interface $f(\mathbf{x}, t)=0$ defined as

$$
2 \kappa(f)=\nabla \cdot\left(\frac{\nabla f}{|\nabla f|}\right),
$$

and $\mathbf{n}=\nabla f /|\nabla f|$ is a unit normal vector.

In Sec. VC, we compare experiments with the solution of Eqs. (2)-(5) in a two-dimensional domain. We also compare with established vibroequilibria theory, which is summarized below.

\section{A. Vibroequilibria theory}

The theory of vibroequilibria [19-21] provides a method for calculating the quasisteady (average) equilibrium states of a fluid configuration with a free interface. It assumes a separation of timescales between the forcing at frequency $\omega$ and the slower (averaged) response of the fluid, characterized by the viscous timescale $L^{2} / v$, and the period of the primary sloshing modes [20,21]; if incompressibility is assumed, then this further requires the forcing frequency to be below the first acoustic resonance.

A variational approach using a time-averaged Lagrangian casts the problem as one of determining critical points for 
a functional that depends on the shape of the interface. For this step, it is further assumed that the leading-order velocity field is potential, with $\mathbf{u}=\nabla \phi$, and varies harmonically at the frequency of the applied forcing:

$$
\phi(\mathbf{x}, t)=v_{\eta} \tilde{\phi}(\mathbf{x}) \cos (\omega t)+\cdots .
$$

The free surface is split into oscillatory and average parts,

$$
f(\mathbf{x}, t)=\widetilde{F}(\mathbf{x})+\tilde{f}(\mathbf{x}) \cos (\omega t)+\cdots .
$$

Note that rotational motion of the container is not generally compatible with potential flow. Of the three rotational contributions in Eq. (3), it is typically the final term (the Euler acceleration) that is the largest for the resonant modes of interest. For these excitations, none of the rotational terms are as large as the linear acceleration $\omega v_{\eta}$, which justifies neglecting them at leading order so that the standard (variational) approach to vibroequilibria theory can be used; the error this introduces is discussed in Sec. VC.

If there is a constant gravitational acceleration $g$ taken to be aligned with the vertical $z$ axis (see Fig. 3), then the vibroequilibria defined by the surface $\tilde{F}(\mathbf{x})=0$ is dynamically stable if it is a local minimum of the time-averaged functional $[20,21]$,

$$
\begin{aligned}
\mathcal{L}= & \int_{\langle\Omega\rangle}\left(g z+\frac{v_{\eta}^{2}}{4}|\nabla \tilde{\phi}|^{2}+P_{0}\right) d \Omega \\
& +\frac{\Gamma}{\rho} \int_{\langle S\rangle} d S-\frac{\Gamma}{\rho} \sin \beta \int_{\langle W\rangle} d W,
\end{aligned}
$$

where $P_{0}$ is a Lagrange multiplier for enforcing conservation of volume. The functional (8) should be minimized over permissible configurations of $\tilde{F}(\mathbf{x})$. Assuming the surface can be expressed as a single-valued function $z=F(x, y)$ and using the rescalings

$$
\mathbf{x} \rightarrow L \mathbf{x}, \quad P_{0} \rightarrow \frac{\Gamma}{\rho L} P_{0}, \quad \widetilde{\phi} \rightarrow L \tilde{\phi}, \quad \mathcal{L} \rightarrow \frac{\Gamma L^{2}}{\rho} \mathcal{L},
$$

the functional can be rewritten (see Ref. [22] for details) as

$$
\begin{aligned}
\mathcal{L}= & P_{0} \mathcal{V}+\int_{S} d S+\eta \int_{S}(\mathbf{e} \cdot \mathbf{x}) q(\mathbf{x}) d S \\
& -\sin \beta \int_{W} d W+\frac{\mathrm{Bo}}{2} \int_{S}\left[F(x, y)^{2}-d^{2}\right] d x d y,
\end{aligned}
$$

where $d$ is the liquid depth with a flat horizontal surface and $q(\mathbf{x})=\partial \widetilde{\phi} / \partial n$. The nondimensional parameter,

$$
\eta=\frac{\rho L}{4 \Gamma} v_{\eta}^{2},
$$

characterizes the ratio of vibrational energy to surface energy, while the Bond number,

$$
\mathrm{Bo}=\frac{\rho L^{2}}{\Gamma} g,
$$

characterizes the relative importance of gravitational and surface energy. We minimize Eq. (10) using the boundary element method, as in Ref. [22], to obtain the predicted vibroequilibria surface.

\section{VIBROEQUILIBRIA IN A CYLINDRICAL CONTAINER}

Figure 4 shows the evolution of the fluid surface in three vibrated cylindrical containers approximately half-filled with silicone oil, to be compared with the unforced reference cell (top row). In each experiment, the free surface is captured from the front and from a lateral view and is illustrated at three different times (labeled in the figure) measured with respect to the beginning of the excitation in microgravity at $t=0 \mathrm{~s}$. These snapshots are selected to show the liquid surface at the beginning of the excitation, at the time when the contact line reaches the upper lid of the container (or its maximum height), and at an intermediate point between these.

The vibrations are delivered at a fixed frequency $f=$ $59.3 \mathrm{~Hz}$, which corresponds to the first resonant mode, and for three applied voltages of 80,90 , and $100 \mathrm{~V}$; these give vertical vibrational velocities $v_{z}$ (measured at the accelerometer) of 73.9 [Figs. 4(a)-4(c)], 84.6 [Figs. 4(d)-4(f)], and $94.8 \mathrm{~mm} / \mathrm{s}$ [Figs. 4(g)-4(i)], respectively. For the experiment shown in Figs. 4(a)-4(c), each snapshot of the vibrated cell is shown below a corresponding snapshot of the reference cell. The front and rear halves of the contact line are highlighted in solid and dashed lines, respectively, as a visual reference. Recall that the lateral views are obtained after reflection, as illustrated in Fig. 2.

The experiments shown in Fig. 4 are quantified in Fig. 5, where the uppermost (solid dots) and lowermost (diamonds) visible points of the surface, which are located along the contact line and in the interior, respectively, are shown as a function of time. The vibrated surface (marked in black) and the reference experiment (marked in gray) can be compared. Each plot is shown above the corresponding residual gravity measurement, given in the airplane reference frame. The vertical vibrational velocities $v_{z}$ measured by the accelerometer of 73.9 (a), 84.6 (b), and $94.8 \mathrm{~mm} / \mathrm{s}$ (c) are indicated, as are the times (shown with vertical lines) of the snapshots included in Fig. 4.

Prior to the beginning of the excitation in microgravity, the equilibrium reflects a balance between residual gravity and contact forces. Since silicone oil easily wets most surfaces and has a low contact angle, the surface resembles a spherical cap; see Figs. 4(a), 4(d) and 4(g). In the absence of applied vibrations, this spherical caplike shape is maintained throughout the experiment, although it responds with some degree of motion to the changing residual gravity, as seen in the snapshots of the reference cell in Figs. 4(a)-4(c). Applied vibrations, in contrast, modify this interface shape significantly.

Immediately after the forcing is initiated, the fluid moves toward a new (quasisteady) equilibrium, evolving on a slow timescale compared to the forcing period. This vibroequilibria effect is quantified by recording the motion of the contact line with respect to the minimum height of the visible surface, which occurs in the interior. What is generally observed with increasing amplitude is the gradual upward motion of the "front" and "rear" halves of the contact line, as seen in Figs. 4 and 5. This movement of the surface, which is driven by the spatially inhomogeneous vibrational velocity field, is affected by the residual gravity level. In particular, there is an evident correlation with the vertical gravity component (black curve 


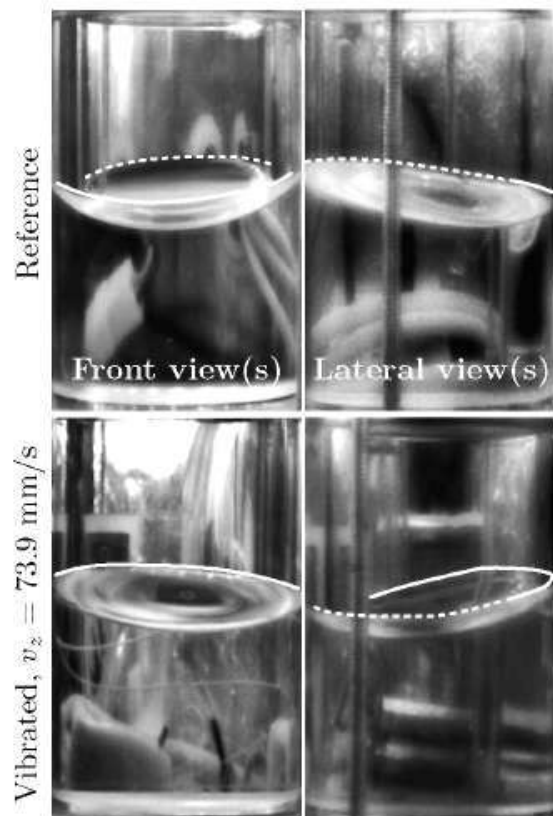

(a) $t=0 \mathrm{~s}$

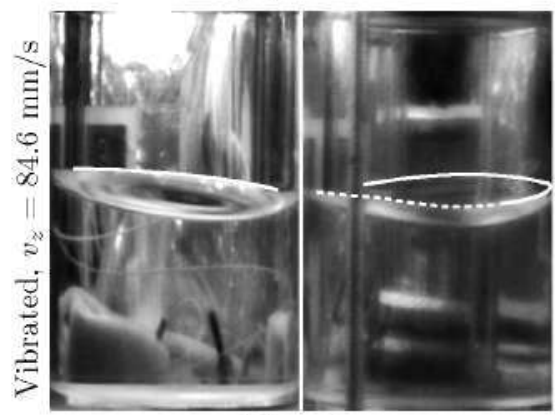

(d) $t=0 \mathrm{~s}$

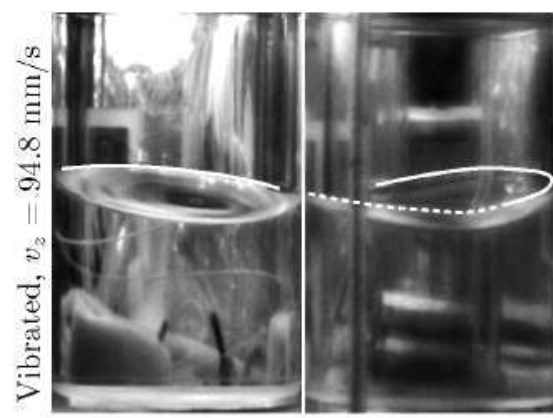

(g) $t=0 \mathrm{~s}$

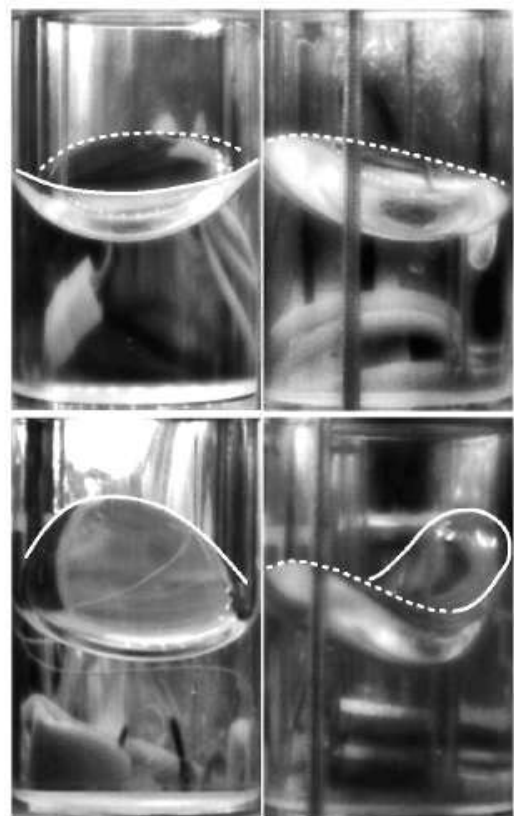

(b) $t=3.475 \mathrm{~s}$

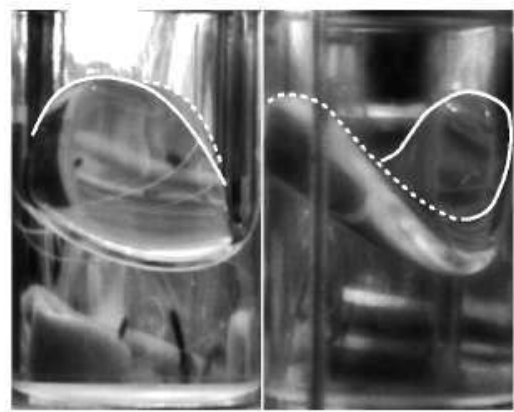

(e) $t=3.488 \mathrm{~s}$

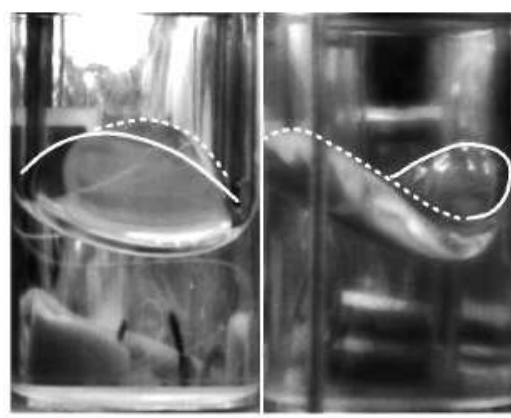

(h) $t=1.517 \mathrm{~s}$

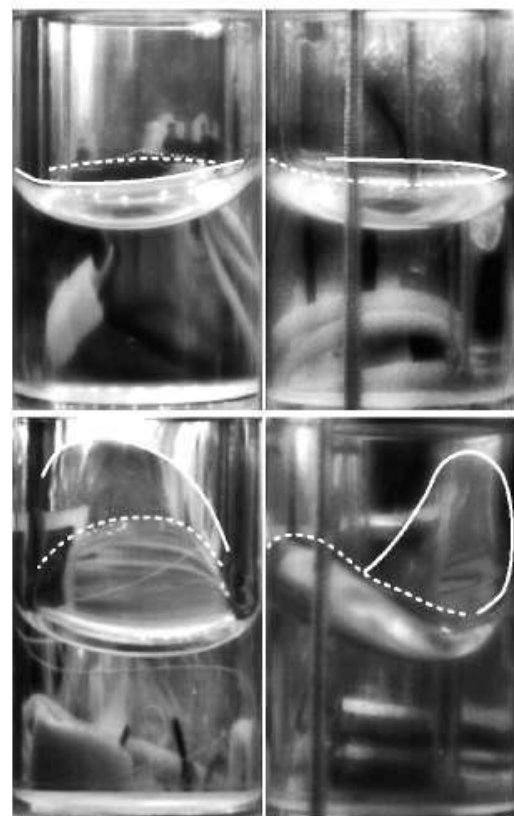

(c) $t=7.640 \mathrm{~s}$

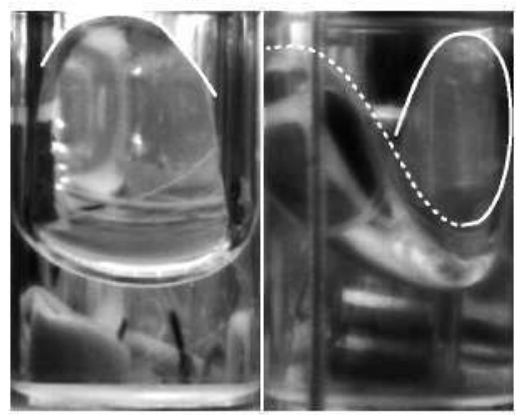

(f) $t=6.381 \mathrm{~s}$

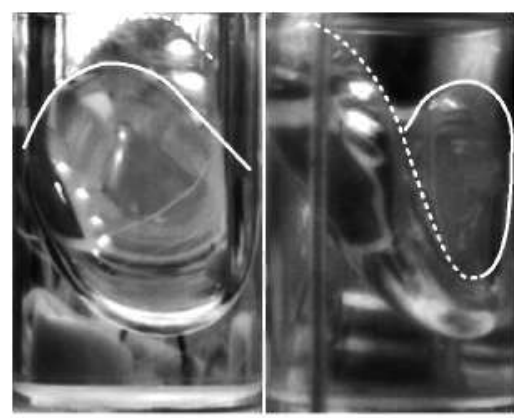

(i) $t=2.444 \mathrm{~s}$

FIG. 4. Snapshots (front and lateral views) showing the evolution of the surface of silicone oil in a cylindrical cell. The upper row of panels (a)-(c) shows the fluid behavior in the reference cell (experiencing residual acceleration of the airplane) at the given times, paired with corresponding snapshots of a vibrated experiment. The vibrated experiments are excited at $f=59.3 \mathrm{~Hz}$ with vibrational velocities [(a)-(c)] $v_{z}=73.9$, [(d)-(f)] 84.6 , and [(g)-(i)] $94.8 \mathrm{~mm} / \mathrm{s}$, which correspond to applied (peak) voltages of 80,90 , and $100 \mathrm{~V}$, respectively. To help visualize the free surface deformation, contact lines at "front" and "rear" halves of the lateral wall are highlighted with solid and dashed lines.

in the lower panels of Fig. 5), reflecting the suppression of vibroequilibria effects for a given vibrational forcing with increasing gravity level (Bond number).

The interior of the free surface, on the other hand, tends to move downward per conservation of mass (see Figs. 4 and 5). Depending on the forcing amplitude, the contact line may reach the upper lid of the container, as with the experiments at 84.6 and $94.8 \mathrm{~mm} / \mathrm{s}$ [Figs. 5(b) and 5(c) and Figs. 4(f) and 4 (i)] or stop prior to that, as with the experiment at $73.9 \mathrm{~mm} / \mathrm{s}$
[Fig. 5(a) and Fig, 4(c)]. For the experiments where the contact line reaches the top of the container, the effect of increasing vibrational amplitude is reflected in the time required for the fluid to arrive at that boundary. This contact time, which decreases from $6.38 \mathrm{~s}$ for $v_{z}=84.6 \mathrm{~mm} / \mathrm{s}$ to $2.44 \mathrm{~s}$ for $v_{z}=94.8 \mathrm{~mm} / \mathrm{s}$, is also affected by the residual gravity level. Generally, the effect of (vertical) gravity is to flatten the surface; a higher vertical gravity level thus requires greater applied forcing to achieve a similar vibroequilibria effect. 